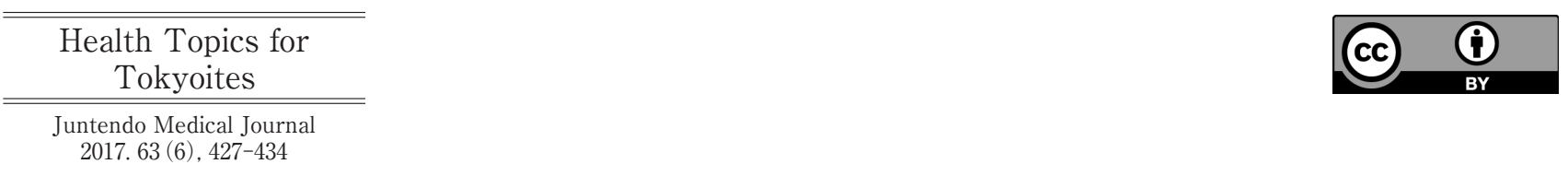

\title{
Current Medical Treatment of Obstructive Sleep Apnea (OSA) in Sleep Center
}

\author{
RYUTARO SHIRAHAMA*1) 2), MANAMI KIMURA*1), HirOO WADA*1), TAKESHI TANIGAWA*1) \\ *1) Department of Public Health, Juntendo University Faculty of Medicine, Tokyo, Japan, \\ *2) RESM Sleep Center, Kanagawa, Japan
}

\begin{abstract}
Key words: sleep disordered breathing, obstructive sleep apnea, traffic accident, cardiovascular disease, mental disorder
\end{abstract}

\section{Introduction}

Obstructive Sleep Apnea (OSA) refers to the occurrences of complete or partial obstruction of the upper airway during sleep. OSA is often associated with intermittent hypoxemia and hypercapnia. Intermittent hypoxemia causes endothelial dysfunction and long-term hypoxic stresses in various organs ${ }^{1)}{ }^{2)}$. For adults, OSA causes sleep deprivation induced symptoms and indirectly induces systemic symptoms, such as impaired glucose tolerance, hypertension, and potentially cause serious traffic accidents ${ }^{3)-9}$. The most important consequence of OSA may be the increased risk for cardiovascular morbidity and mortality.

\section{Functions of sleep and types of sleep disorders}

Generally, older populations tend to require shorter sleep duration. For example, young adults need about 8 hours of sleep, but elderly adults only need about 6 hours of sleep. The percentage of REM sleep changes by age. The percentage of REM sleep of an infant is about $50 \%$, but after childhood it changes to about $20 \%$. Some of the functions of REM sleep is to relieve stress and fix memory; the functions of non-REM sleep include the secretion of growth hormone, decrease core body temperature, and improve immunologic functions.

There are many types of sleep disorders as classified in the most recent edition of the International Classification of Sleep Disorders (ICSD), ICSD-III, such as insomnia disorder, sleep-disordered breathing (SDB), central disorders of hyper somnolence, parasomnias, sleep related movement disorders, and circadian rhythm sleep-wake disorders. And OSA is one of the main diseases of SDB.

\section{Characteristics of OSA}

\section{Risk factors for OSA}

Intermittent upper airway obstruction causes OSA. Specifically, common factors of OSA include obesity, specific craniofacial disorders (e.g., Treacher-Collins, Pierre-Robin syndromes), retroposed mandible/maxillae, adenotonsillar hypertrophy, nasal problems (e.g. septal deviation), allergic rhinitis, endocrine abnormalities (e.g. hypothyroidism/acromegaly), polycystic ovarian syndrome, post menopause, down syndrome, family aggregation,

Corresponding author: Ryutaro Shirahama

RESM Sleep Center

The Maruhachi Shin-Yokohama building $4 \mathrm{~F}$

3-8-12 Shinyokohama, Kouhoku-ku, Yokohama-shi, Kanagawa 222-0033, Japan

TEL: +81-45-475-5155 E-mail: MD.shirahama@live.jp

39th Health Topics for Tokyoites: Sleep Apnea Syndrome, the Important Disorder for Both Adults and Children: Have a Healthy and Safety Life Without Snoring [Held on Feb. 18, 2017〕

〔Received Aug. 7, 2017〕〔Accepted Oct. 31, 2017〕

Copyright (C) 2017 The Juntendo Medical Society. This is an open access article distributed under the terms of Creative Commons Attribution License (CC BY), which permits unrestricted use, distribution, and reproduction in any medium, provided the original source is properly credited. doi: $10.14789 /$ jmj .63 .427 
and the APOع4 allele (in subjects $<65$ yr. ${ }^{10)}$.

\section{Consequences of OSA}

Nocturnal symptoms of obstructive sleep apnea syndrome (OSAS) are disrupted sleep due to snoring and pauses in breathing caused by OSA. Hypoxemia, hypercapnia, pleural pressure variation, and occurrence of arousal response are caused by the OSA. Diurnal symptoms of OSAS are excessive daytime sleepiness, sleep attack, impaired vigilance, and psychomotor deficiency. OSA adversely affects metabolic issues, cardiovascular

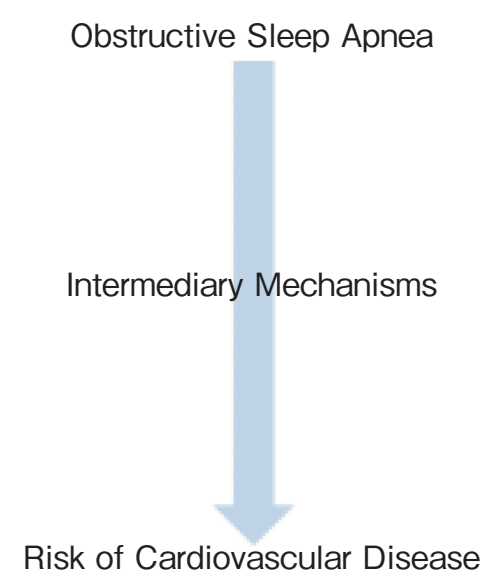

Abu S.M. Shamsuzzaman et al ; JAMA, $2003 ; 290: 1906$ disease (CVD), diabetes, stroke, cognitive issues, and decreases life expectancy.

The risk of cardiovascular disease increased in patients with Respiratory disorder index $(\mathrm{RDI}) \geqq$ 11 , and it was indicated that the odds ratio was (1.27-2.38) for coronary artery disease ${ }^{11)}$. Many mechanisms are related to cardiovascular diseases and OSA (Figure-1).

In recent years, the focus seems to be on the influence of sleep disorders and psychotic disorders. We investigated the relationship between OSA and psychotic disorders in our sleep center. Of the 970

Figure-1 Intermediary mechanisms associated with OSA

\author{
Hypoxemia \\ Reoxygenation \\ Hypercapnia \\ Intrathoracic Pressure Changes \\ Arousals \\ Sympathetic Activation \\ Endothelial Dysfunction \\ Vascular Oxidative Stress \\ Inflammation \\ Increased Coagulation \\ Metabolic Dysregulation \\ Hypertension \\ Congestive Heart Failure \\ Cardiac Arrhythmia \\ Cardiac Ischemia \\ Cerebrovascular Disease
}

Mean age 51.8 years Mean AHI 45.5 times per hour



Figure-2 Mental disorder and OSA in sleep center (24 people) 


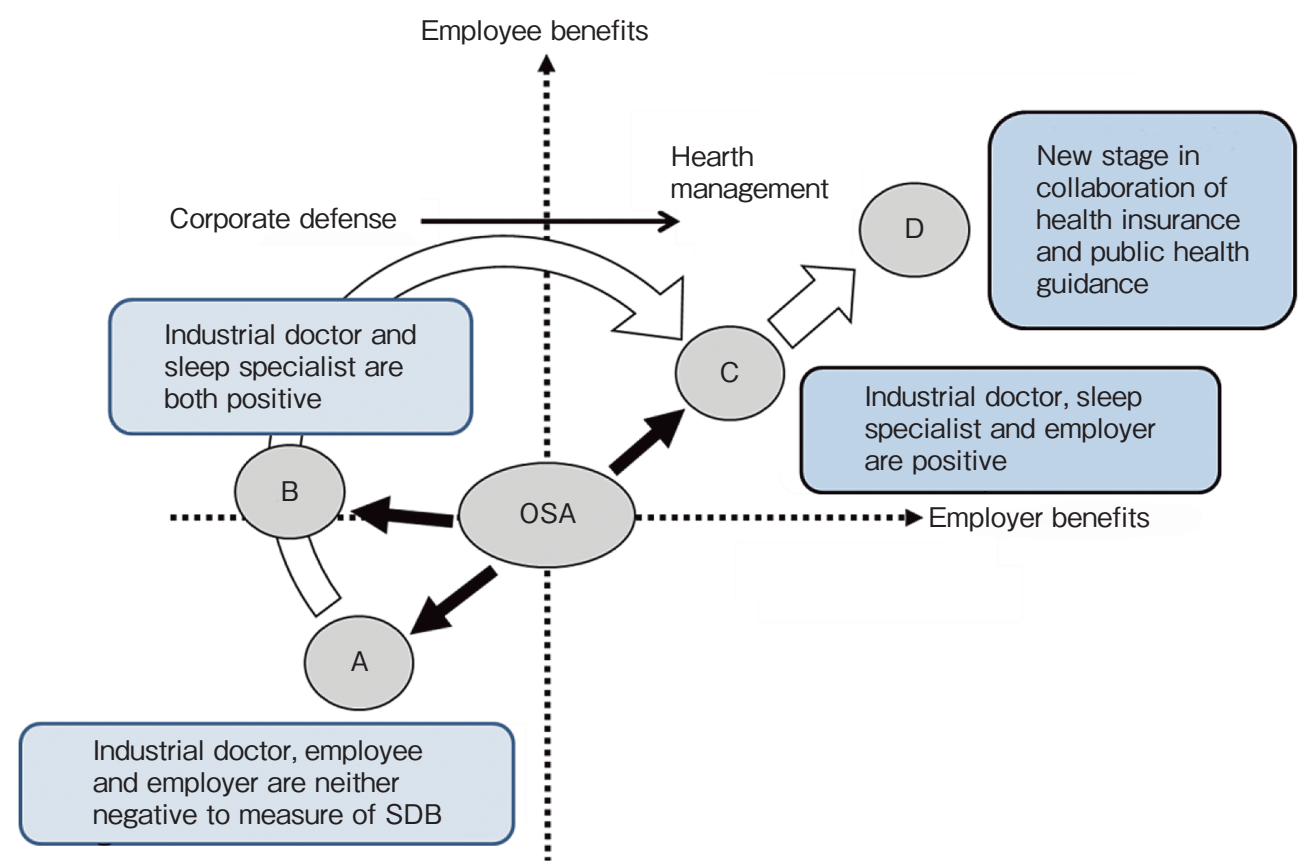

Figure-3 Staging of changing the motivation of management for OSA Alteration from the ministry of health, labour and welfare workers' accident disease clinical study (2016).

people who were diagnosed as OSA with polysomnography in our center, 24 people were diagnosed as psychotic disorder, 83\% with depression, 5\% with borderline disorder, $4 \%$ with adjustment disorder, $4 \%$ with schizophrenia, and $4 \%$ with anxiety neurosis (Figure-2).

\section{OSA and traffic accidents}

OSA prevalence is about $13 \%$ of men in the general population of USA and at least $20-25 \%$ in the commercial driving population. Commercial truck accidents cause about 4,000 deaths and 80,000 serious injuries yearly in the US. In Japan, a highway bus crash in April 2012, who died or were injured of the 46 people. A medical investigation from the crash revealed that the driver had moderate SDB. Another bus crush in May 2015, resulted in 17 people suffering injuries. Similar to the accident in April 2012, the medical investigation report found that the main reason of the accident was driver falling asleep due to OSA.

OSA is primary medical cause of excessive daytime sleepiness (EDS) and increases the risk of accidents 2.5 times $^{7)}$.

Early discovery and early treatment of OSA are needed to reduce traffic accidents and save lives as crash risk is reduced by $72 \%$ following treatment with Continuous Positive Airway Pressure (CPAP). So, collaboration of commercial truck companies, industrial doctors, and sleep specialists is very important.

We are now going to establish guidelines for this collaboration. Stages of the changing the approach of management for OSA are shown (Figure-3).

\section{Treatment with OSA}

Treatment of OSA ranges from general life style improvements, CPAP, Oral appliances (OA), and surgical treatment.

Some general life style improvements include losing weight, restriction of drinking before going to bed, restriction of drinking alcohol, cessation of smoking, and lateral recumbent position sleep.

CPAP treatment increases deep sleep and improved the blood levels of oxygen to normal (Figure-4). Treatment with CPAP leads to lowering high blood pressure ${ }^{12)}$, lowering the risk of coronary artery disease ${ }^{13)}$, and heart failure ${ }^{14)}$.

\section{Case reports}

\section{OSA and depression case (patient A)}

This is a 47-year old man whose chief complaints were daytime sleepiness, snoring, awaking during 
nocturnal sleep, frequent urination at night, and lack of deep sleep. He had a history of lipid disorders, obesity, and depression. Full polysomnography findings are shown in Table- 1 , Figure -5 and Figure-6 (Apnea Hypopnea Index (AHI) was 51.7 per hour).
He was diagnosed with severe OSAS and was put on CPAP (Sleepmate S9 Auto, Resmed, Australia). After CPAP treatment, his AHI dropped to 2.0 times per hour and the previous complaints of daily sleepiness, snoring, wake time after sleep onset (WASO), frequent urination at night and lack of

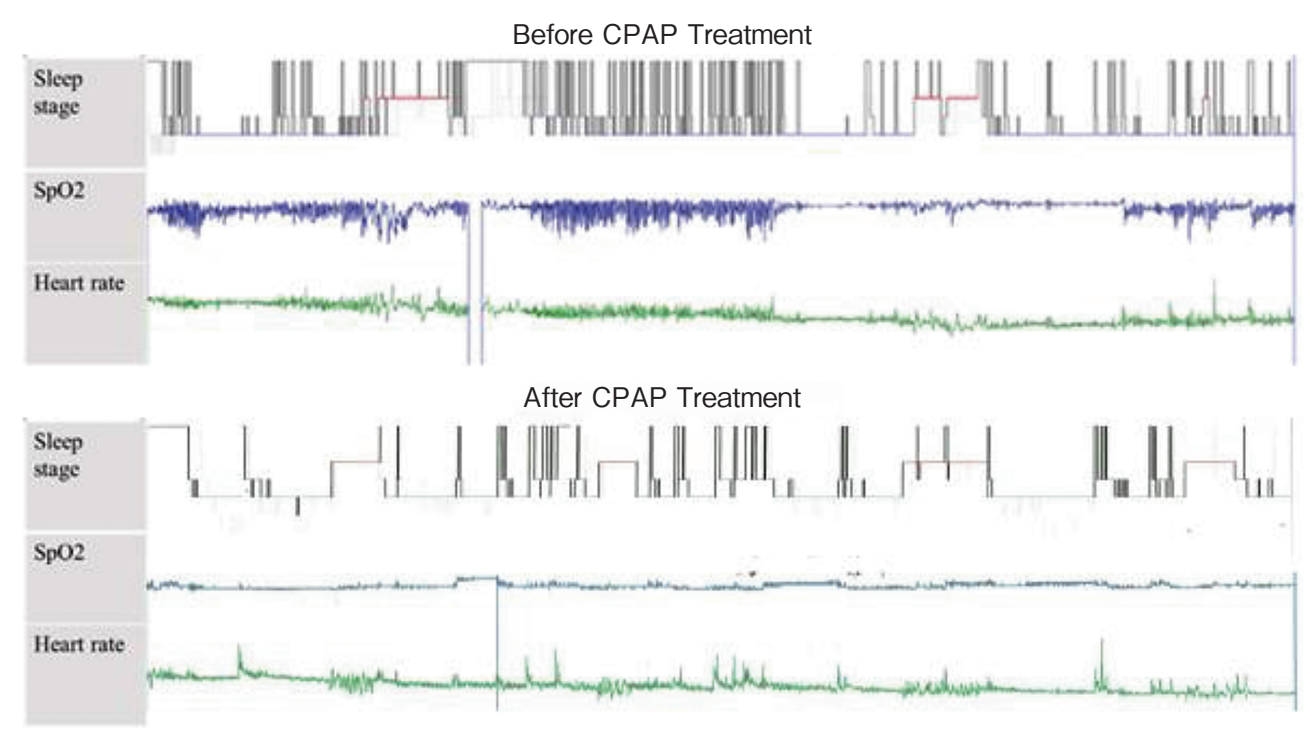

Figure -4 Changes of Sleep stages and oxygen levels

Table-1 Polysomnography analysis data of patient A

\begin{tabular}{|c|c|c|}
\hline Time in bed TIB : Light off $\rightarrow$ Light on & 485.0 & $\min$ \\
\hline Sleep period time SPT : Sleep onset $\rightarrow$ Last sleep page & 478.7 & $\min$ \\
\hline Total sleep time TST : SPT-WASO & 388.7 & $\min$ \\
\hline Wake time after sleep onset WASO : SPT-TST & 90.0 & $\min$ \\
\hline Sleep efficiency : TST/TIB $\times 100 \%$ & 80.1 & $\%$ \\
\hline SIeep latency & 6.3 & $\min$ \\
\hline REM latency & 85.5 & $\min$ \\
\hline Sleep stage REM (\%TST) & 13.6 & $\%$ \\
\hline Sleep stage N1 (\%TST) & 19.2 & $\%$ \\
\hline Sleep stage N2 (\%TST) & 67.2 & $\%$ \\
\hline Sleep stage N3 (\%TST) & 0.0 & $\%$ \\
\hline Arousal index & 39.7 & \#/h of TST \\
\hline Apnea Hypopnea index : AHI & 51.7 & $\# / \mathrm{h}$ of $\mathrm{TST}$ \\
\hline Apnea index AI & 1.4 & $\# / \mathrm{h}$ of TST \\
\hline Hypopnea index HI & 50.3 & $\# / \mathrm{h}$ of $\mathrm{TST}$ \\
\hline $3 \%$ Oxygen desatumtion index & 48.9 & $\# / \mathrm{h}$ of $\mathrm{TST}$ \\
\hline $\mathrm{SpO} 290 \%>$ & 19.7 & $\min$ \\
\hline Minimum of the resp. events $\mathrm{SpO} 2 \mathrm{~min}$ levels & 81 & $\%$ \\
\hline Total duration with snoring (\%TST) & 11.3 & $\%$ \\
\hline Periodic limb movement of sleep PLMS index & & \#/h of TST \\
\hline Arousal meeting PLMS criteria & & $\# / \mathrm{h}$ of TST \\
\hline
\end{tabular}




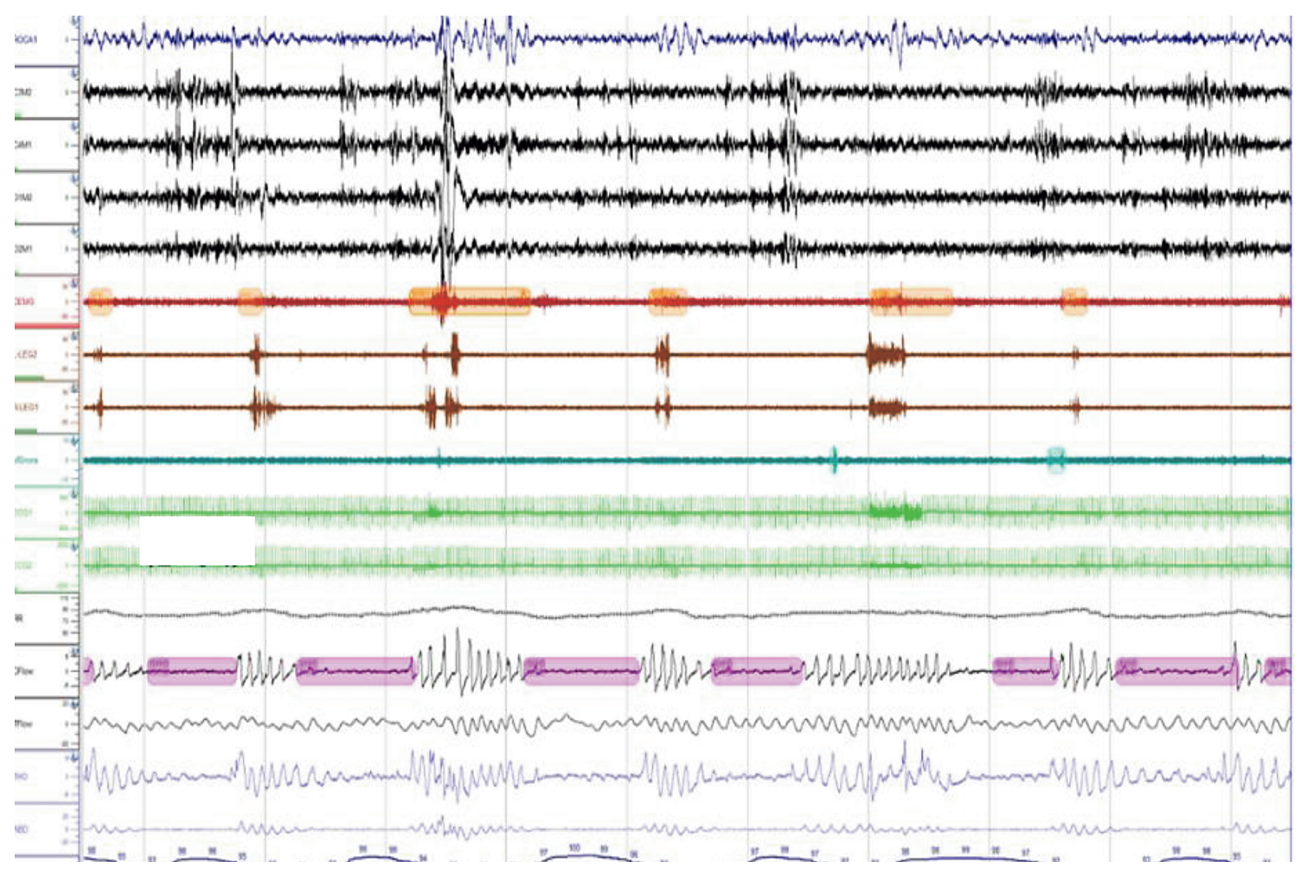

Figure-5 Polysomnography data of patient A (five minutes)

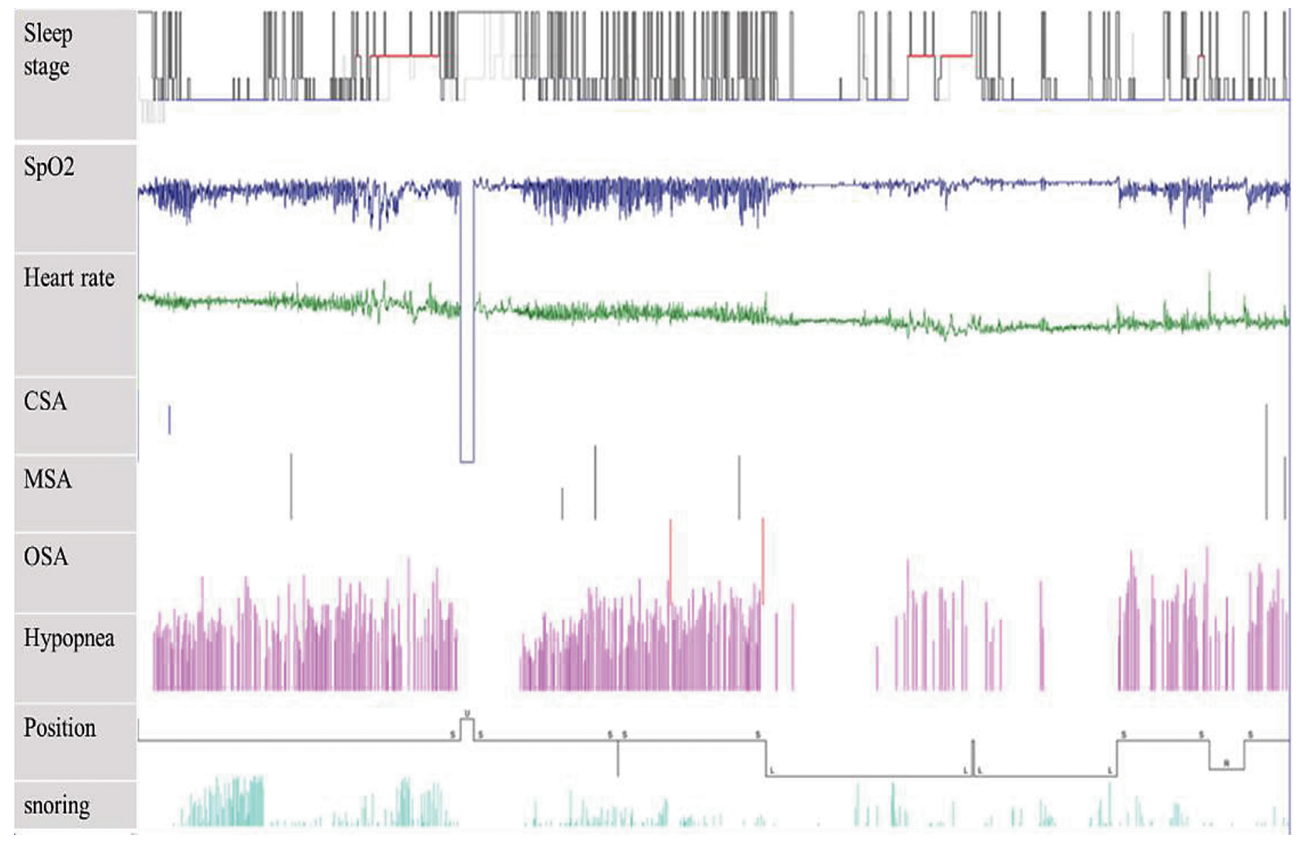

Figure-6 Polysomnography data of patient A (overnight)

deep sleep disappeared; the CES-D scale also improved.

\section{OSA and diabetes case (patient B)}

This is a 56-year old man whose chief complaints were daytime sleepiness, snoring, awakening during nocturnal sleep, frequent urination at night, and lack of deep sleep. He also had a history of diabetes. His HbAlc blood test result was 9.2\% (normal range of $\mathrm{HbAlC}$ in Japan ranges from $4.3 \%$ to $5.8 \%)$. Full polysomnography findings are shown in Table-2, Figure-7, and Figure-8.

The Apnea Hypopnea Index (AHI) was 36.3 times per hour. He was diagnosed with severe 
Table-2 Polysomnography analysis data of the patient B

\begin{tabular}{lcc}
\hline Time in bed TIB : Light off $\rightarrow$ Light on & 479.8 & min \\
Sleep period time SPT : Sleep onset $\rightarrow$ Last sleep page & 476.4 & min \\
Total sleep time TST : SPT-WASO & 411.4 & min \\
Wake time after sleep onset WASO : SPT-TST & 65.0 & min \\
Sleep efridency : TST/TIB × 100\% & 85.7 & $\%$ \\
SIeep latency & 3.4 & min \\
REM latency & 124.5 & min \\
Sleep stage REM (\%TST) & 20.2 & $\%$ \\
Sleep stage N1 (\%TST) & 23.6 & $\%$ \\
Sleep stage N2 (\%TST) & 56.3 & $\%$ \\
Sleep stage N3 (\%TST) & 0.0 & $\%$ \\
& & \\
Arousal index & 41.6 & \#/h of TST \\
Apnea Hypopnea index : AHI & 36.3 & \#/h of TST \\
Apnea index AI & 31.9 & \#/h of TST \\
Hypopnea index HI & 4.4 & \#/h of TST \\
3\%Oxygen desatumtion index & 31.5 & \#/h of TST \\
SpO2 90\%> & 54.6 & min \\
Minimum of the resp. events SpO2 min levels & 77 & $\%$ \\
Total duration with snoring (\%TST) & 17.2 & $\%$ \\
Periodic limb movement of sleep PLMS index & 12.4 & \#/h of TST \\
Arousal meeting PLMS criteria & 1.0 & \#/h of TST \\
\hline
\end{tabular}

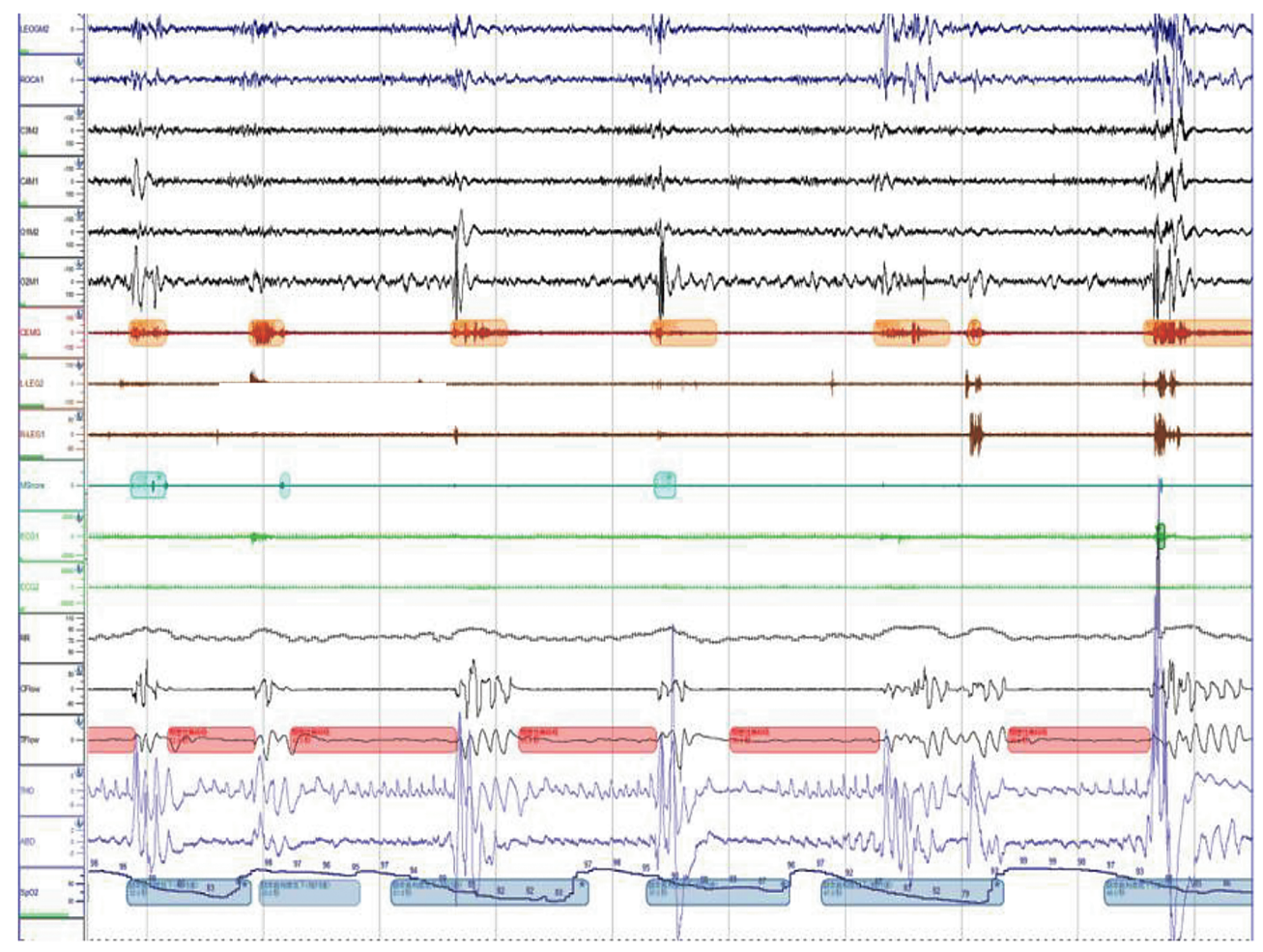

Figure-7 Polysomnography data of patient B (five minutes) 


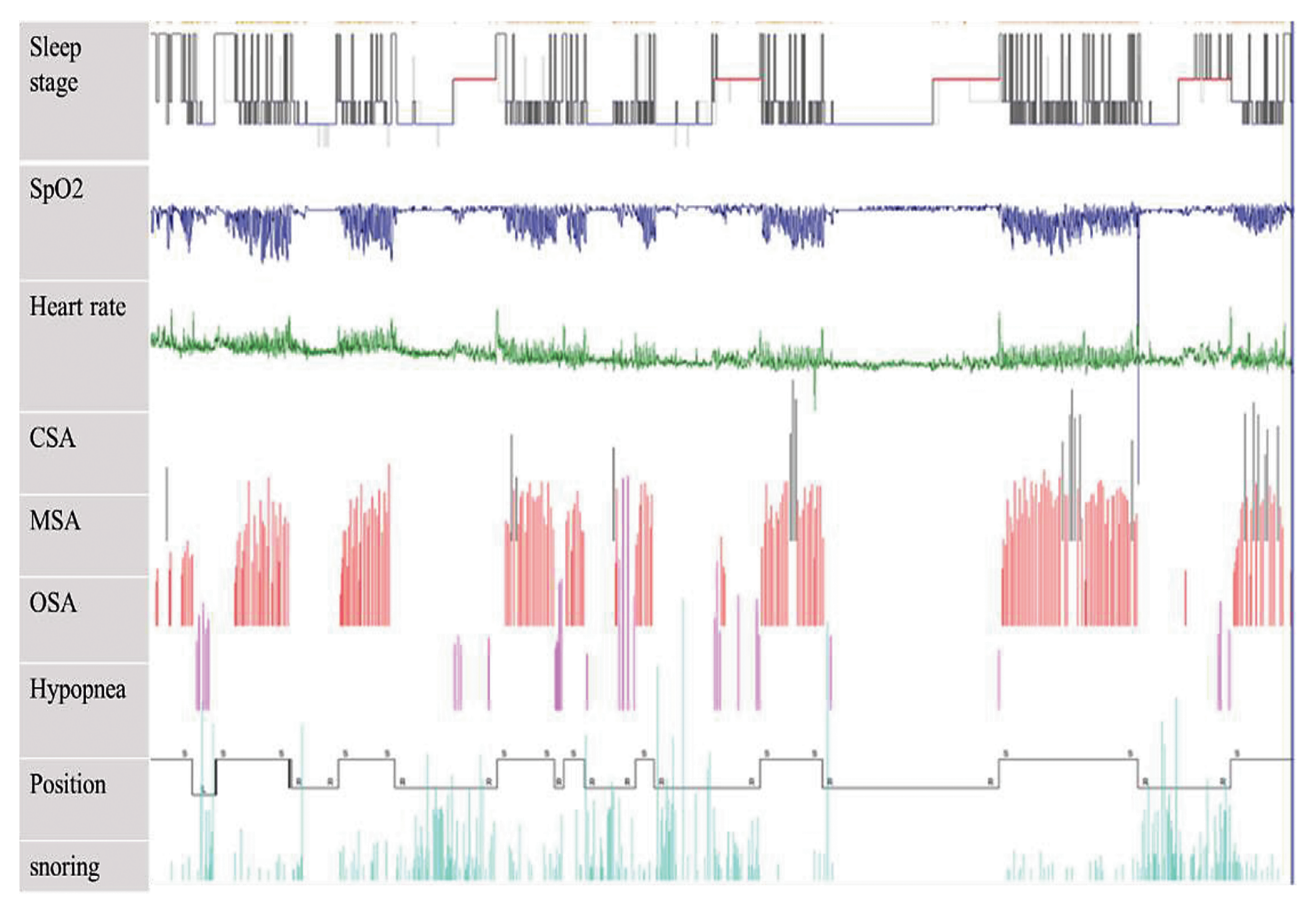

Figure-8 Polysomnography data of patient B (overnight)

OSAS and was put on CPAP (REMstar Auto System One 60, Philips, Netherlands).

After CPAP treatment, his AHI dropped to 1.0 times per hour and the previous complaints of daily sleepiness, snoring, awaking nocturnal sleep, frequent urination at night, and lack of deep sleep disappeared; BMI also improved from 25.7 to 24.7 and $\mathrm{HbA1c}$ levels dropped to $6.4 \%$.

\section{OSA and changes of disease severity from weight loss case}

This is a 41-year old man whose chief complaints were daytime sleepiness, snoring, and lack of deep sleep. Utilizing full polysomnography, his AHI was reported as 36.1 times per hour. He was diagnosed with severe OSAS and was put on CPAP (Sleepmate S9 Auto, Resmed, Australia). He managed to lose 10 kilograms and also received nose mucous membrane cauterization, fexofenadine, and mometasone furoate hydrate. After weight loss and EMT treatment, his AHI dropped to 8.8 times per hour via the home sleep test; However, his AHI dropped to 4.4 times per hour after being treated with OA.

\section{Conclusion}

OSA is associated with intermittent hypoxemia and hypercapnia. Intermittent hypoxemia causes endothelial dysfunction and long-term hypoxic stresses in various organs. OSA may increase the risk for the development of various diseases such as hypertension, diabetes, depression, cardiovascular disease and cause serious traffic accidents.

Therefore, the need to be tested promptly and undergo specialized medical consultation if snoring, daytime sleepiness, concentration and frequent urination continue to interrupt one's daily life is paramount.

\section{References}

1) Ip MS, Tse HF, Lam B, Tsang KW, Lam WK: Endothelial function in obstructive sleep apnea and response to treatment. Am J Respir Crit Care Med, 2004; 169: 348-353.

2) Lavie L: Oxidative stress: a unifying paradigm in obstructive sleep apnea and comorbidities. Prog Cardiovasc Dis, 2009; 51: 303-312.

3) Ip MS, Lam B, Ng MM, Lam WK, Tsang KW, Lam KS: Obstructive sleep apnea is independently associated with insulin resistance. Am J Respir Crit Care Med, 2002; 165: 670-676.

4) Polotsky VY, Patil SP, Savransky V, et al: Obstructive sleep apnea, insulin resistance, and steatohepatitis in severe obesity. Am J Respir Crit Care Med, 2009; 179: 228-234. 
5) Brooks D, Horner RL, Kozar LF, Render-Teixeira CL, Phillipson EA: Obstructive sleep apnea as a cause of systemic hypertension. Evidence from a canine model. J Clin Invest, 1997; 99: 106-109.

6) Lavie P, Herer P, Hoffstein V: Obstructive sleep apnoea syndrome as a risk factor for hypertension: population study. BMJ, 2000; 320: 479-482.

7) Tregear S, Reston J, Schoelles K, Phillips B: Obstructive sleep apnea and risk of motor vehicle crash: systematic review and meta-analysis. J Clin Sleep Med, 2009; 5: 573-581.

8) Burks SV, Anderson JE, Bombyk M, et al: Nonadherence with employer-mandated sleep apnea treatment and increased risk of serious truck crashes. Sleep, 2016; 39: 967-975.

9) Garbarino S, Guglielmi O, Sanna A, Mancardi GL, Magnavita N: Risk of occupational accidents in workers with obstructive sleep apnea: systematic review and meta-analysis. Sleep, 2016; 39: 1211-1218.
10) Pack AI: Advances in sleep-disordered breathing. Am J Respir Crit Care Med, 2006; 173: 7-15.

11) Shahar E, Whitney CW, Redline S, et al: Sleep-disordered breathing and cardiovascular disease: crosssectional results of the Sleep Heart Health Study. Am J Respir Crit Care Med, 2001; 163: 19-25.

12) Martínez-García MA, Capote F, Campos-Rodríguez F, et al: Effect of CPAP on blood pressure in patients with obstructive sleep apnea and resistant hypertension: the HIPARCO randomized clinical trial. JAMA, 2013; 310: 2407-2415.

13) Garcia-Rio F, Alonso-Fernández A, Armada E, et al: CPAP effect on recurrent episodes in patients with sleep apnea and myocardial infarction. Int J Cardiol, 2013; 168: 1328-1335.

14) Javagheri S, Caref EB, Chen E, Tong KB, AbrahamWT: Sleep apnea testing and outcomes in a large cohort of medicare beneficiaries with newly diagnosed heart failure. Am J Respir Crit Care Med, 2011; 183: 539-546. 